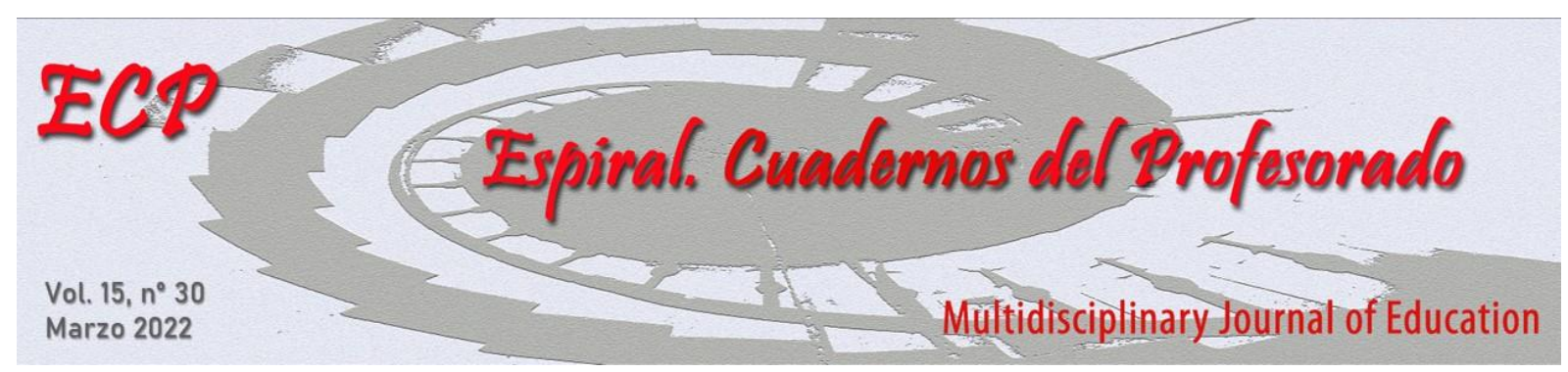

\title{
Impacto de una experiencia Flipped Classroom en las percepciones sobre el aprendizaje del alumnado de Ciencias de la Actividad Física y el Deporte
}

\section{Impact of a Flipped Classroom experience on perceptions of student's Physical Activity and Sport Science learning}

\author{
Ricardo Martín-Moya, Augusto Hoyo-Guillot, Encarnación Ruiz-Montero, \\ y Pedro Jesús Ruiz-Montero \\ Universidad de Granada, España
}

\section{Resumen}

A pesar de la popularidad del aula invertida, su efectividad para lograr un mayor compromiso y resultados de aprendizaje carece actualmente de evidencia empírica sustancial. Este estudio analizó las aportaciones de 54 estudiantes que participaron en un entorno de enseñanza de aula invertida en el Doble Grado en Ciencias de la Actividad Física y del Deporte de la Universidad de Granada (Campus de Melilla), concretamente en la asignatura de Biomecánica del Movimiento Humano. En general, los estudiantes informan una experiencia de aprendizaje más positiva y una mayor participación en el aula invertida. Se seleccionó un estudio con enfoque cualitativo partiendo de las aportaciones del alumnado a través de un diario reflexivo cumplimentado a lo largo de todo el semestre y entrevistas semiestructuradas. El análisis reveló seis categorías que los estudiantes destacan como especialmente propicias para su aprendizaje; compromiso con los compañeros, ser reconocido, relación con el profesor, entorno de aprendizaje físico, aprender con compañeros y usar videos para aprender nuevos contenidos. Los resultados indican que la dimensión afectiva de la participación de los estudiantes es particularmente prominente cuando los estudiantes reflexionan sobre el aprendizaje en el aula invertida.

Palabras clave: aula invertida; aprendizaje; investigación cualitativa; educación superior.

\begin{abstract}
Despite the popularity of the flipped classroom, its effectiveness in achieving greater engagement and learning outcomes currently lacks substantial empirical evidence. This study analyzed the contributions of 54 students who participated in an inverted teaching environment in the Double Degree in Physical Activity and Sports Sciences (Melilla, University of Granada), specifically in the subject of Biomechanics of Human Movement. A study with a qualitative approach was selected based on the contributions of the students through a reflective diary completed throughout the semester and semi-structured interviews. In general, students reported a more positive learning experience and greater participation in the flipped classroom. The analysis revealed six categories that students highlight as especially conducive to their learning; engagement with peers, being recognized, relationship with the teacher, physical learning environment, learning with peers and using videos to learn new content. The results indicate that the affective dimension of student participation is particularly prominent when students reflect on learning in the flipped classroom.
\end{abstract}

Keywords: flipped classroom; learning; qualitative research; higher education

Fecha de recepción: 20/12/2021

Fecha de aceptación: 02/02/2022

Correspondencia: Ricardo Martín-Moya, 1Departamento de Educación Física y Deportiva. Facultad de Ciencias de la Educación y del Deporte de Melilla, Universidad de Granada. Melilla, España Email: rmartinm@ugr.es 
Impacto de una experiencia Flipped Classroom en las percepciones sobre el aprendizaje del alumnado de Ciencias de la Actividad Física y el Deporte

\section{Introducción}

Hoy en día, el papel de los docentes no es solo "enseñar" los conocimientos que tendrán una duración limitada y siempre podrán ser accesibles por otros medios, sino ayudar a los estudiantes a "aprender a aprender" de forma autónoma (Monereo y Alvarez, 2013). Para llevar a cabo una buena formación del profesorado se deben utilizar herramientas didácticas específicas para que la formación sea completa y eficaz. Un gran problema surge en la actualidad cuando se pide al profesorado que adopte un enfoque radicalmente diferente al modelo que ha imperado hasta ahora en las universidades españolas. Deben adaptarse a un modelo de estudiantes protagonistas, estructurando los cursos en torno a la adquisición de competencias en lugar de objetivos, y asumiendo una recreación de modelos pedagógicos a favor de enfoques alternativos e innovadores para la enseñanza (Casey, 2014; Landi et al., 2016). Así, los modelos pedagógicos no reemplazan los estilos de enseñanza, sino que los incorporan a sus estructuras, con enfoques de aprendizaje a largo plazo, centrados en el estudiante. Enseñar a través de nuevas metodologías significa adoptar enfoques que potencien la implicación del alumnado para hacer que el aprendizaje sea más interesante, emocionante y eficaz (Wijayati et al., 2019). Según Assmann (2002), el verdadero cambio en la enseñanza es la unión del aprendizaje y la motivación. Esta unión hará que el proceso de enseñanza-aprendizaje sea más ameno y atractivo, además de mucho más efectivo (HiguerasRodríguez et al., 2020).

En el método de enseñanza tradicional basado en clases magistrales expositivas, el tiempo de clase generalmente se dedica a impartir el contenido del plan de estudios en lugar de involucrar a los estudiantes en ejercicios de aprendizaje activo (Almanasef et al., 2020). En estos modelos, la evaluación suele ser sinónimo de examen y calificación. Esta es una tradición que parece estar cambiando, en la que el papel del alumnado ha cobrado mayor importancia, situándose en el centro del proceso de aprendizaje mediante la evaluación formativa (López et al., 2020). Teniendo en cuenta la formación inicial del profesorado, el uso y desarrollo de sistemas de evaluación formativa en diferentes disciplinas y la promoción de la participación del alumnado en los sistemas y procesos de evaluación son doblemente importantes (Gallardo Fuentes et al., 2018). La primera razón es universal: este tipo de sistema generalmente produce un mejor proceso de aprendizaje y desarrolla una mayor capacidad de aprendizaje permanente (aprender a aprender). La segunda es específica de la formación inicial y de especial relevancia: si los docentes no viven y experimentan su formación inicial, difícilmente podrán poner en práctica sistemas y procesos de evaluación formativa y compartida en el futuro. Esto es aún más importante cuando el sistema educativo actual está enfocado al desarrollo de competencias profesionales (Aranda et al., 2018).

El "aprendizaje activo" se describe como un enfoque de enseñanza que requiere que los estudiantes participen en actividades de clase que han sido diseñadas por los docentes (Bretón et al., 2021). Investigaciones anteriores sugieren que el aprendizaje activo está relacionado con la mejora de los resultados de aprendizaje de los estudiantes e implica un mayor conocimiento del contenido, habilidades interpersonales, pensamiento crítico y habilidades para resolver problemas, así como una mejora de las actitudes hacia el aprendizaje (Fidalgo-Blanco et al., 2019; Jdaitawi, 2020).

En este contexto, las experiencias educativas recientes en educación superior han enfatizado su desarrollo sobre nuevas formas de enseñanza centradas en el alumnado. El aula invertida o Flipped Classroom es un ejemplo de este cambio en el que los profesores y los estudiantes han asumido nuevos roles. Jeong et al. (2021), definen ampliamente el aula invertida como un conjunto de enfoques pedagógicos que trasladan la enseñanza de la transmisión de información fuera de la clase, utilizan el tiempo de clase para el aprendizaje activo y social y requieren que los estudiantes completen actividades previas y posteriores. actividades de clase para beneficiarse plenamente del trabajo en clase (Awidi y Paynter, 2019). La impartición del contenido del curso es posible en la mayoría de los casos gracias a innovaciones tecnológicas como las conferencias en vídeo, que se combinan con el aprendizaje activo en el aula diseñado para crear interacciones significativas entre el alumno y el instructor y entre alumno y alumno (McNally et al., 2017). El aula invertida como se define anteriormente se entiende mejor como un marco pedagógico que contiene una amplia gama de implementaciones diferentes, tanto con respecto a las actividades fuera de clase como dentro de la clase (Matsumoto, 2016). 
Como indicador indirecto de interés y posible prevalencia de implementación, la popularidad del término de búsqueda web "aula invertida", que se observó por primera vez en mayo de 2011, se encuentra actualmente en su nivel más alto y se prevé que aumente (Canales-Ronda y Hernández-Fernández, 2019). Los países que más buscan este término son Suecia, EE. UU., Australia, Canadá y España (McNally et al., 2017). A pesar de esta aparente popularidad, sigue habiendo evidencia empírica insustancial que ilustra la efectividad del aula invertida para lograr un mayor compromiso y resultados de aprendizaje (Abeysekera y Dawson, 2015; Bishop, 2013).

Los elementos clave que constituyen una metodología basada en el aula invertida incluyen (a) La primera fase del aprendizaje es la fase de aprendizaje previa a la clase. El alumnado adquiere conocimientos básicos de la materia al ver los materiales de aprendizaje proporcionados por el profesor en varios formatos, como videos en línea, podcasts o materiales en formato de texto, antes de la clase (SteenUtheim y Foldnes, 2018), un incentivo para que los estudiantes se preparen para la clase (por ejemplo, pruebas previas a la clase), y un mecanismo para evaluar la comprensión del estudiante (Ej., pruebas graduadas previas a la clase) y (b) la segunda fase del aprendizaje en el aula invertida es la fase de aprendizaje en clase. En esta fase, los estudiantes tienen actividades de aprendizaje activo centradas en el trabajo estudiante en clase, como conferencias interactivas, resolución de problemas, experimentos de laboratorio, juego de roles y diseño y creación de espacios colaborativos (Hermsen et al., 2019; van Alten et al., 2020).

Los profesores en entornos de educación superior pueden preferir el aula invertida por diversas razones. Una ventaja importante de dicha metodología es que brinda a los estudiantes la capacidad de estudiar a su propio ritmo y en su propio tiempo (Davies et al., 2013); también mejoran el tiempo útil de práctica durante las clases (O'Flaherty y Phillips, 2015). Estudios anteriores han encontrado que el método de enseñanza de aula invertida disminuye el estrés (Marlowe, 2012) y aumenta la cooperación, la innovación, la orientación a la tarea y las habilidades de metacognición de los estudiantes (Strayer, 2012). Sin embargo, el número y la amplitud de los estudios sobre el método del aula invertida indican que la investigación sobre este tema aún está en barbecho. Además, el impacto del aula invertida todavía se discute. Por ejemplo, varios estudios han encontrado que el método de aula invertida aumenta el rendimiento de los estudiantes (Baepler et al., 2014; Hung, 2015; Moravec et al., 2010; Murphree, 2014; Yoon et al., 2020). Por el contrario, diversos estudios han encontrado que las aulas invertidas no tienen ningún impacto en la mejora del rendimiento (Bachnak y Maldonado, 2014; Bishop, 2013; Howell, 2013; Jeong et al., 2021; McLaughlin et al., 2013). Estas diferencias pueden estar relacionadas con los tipos y formas de implementación. El aula invertida es una nueva cultura para estudiantes y profesores y no tiene un marco teórico estricto, lo que conduce a resultados variables. Otro factor que podría explicar estas diferencias está relacionado con la motivación de los estudiantes (Chen, 2021).

En el estudio actual, se investigaron los efectos de la metodología de aula invertida sobre los logros de aprendizaje de los estudiantes en una asignatura del Doble Grado en Ciencias de la Actividad Física y del Deporte de la Universidad de Granada (Campus de Melilla). Es importante investigar la eficiencia de estas metodologías en relación con las percepciones de los estudiantes. Estos resultados ofrecerán nuevos resultados para el aprendizaje en aula invertida en investigadores y profesores. Por lo tanto, el objetivo del presente estudio es el de conocer los niveles de satisfacción y adquisición de competencias por parte del alumnado tras la inmersión en una experiencia de enseñanza-aprendizaje basada en la metodología de aula invertida.

\section{Método}

Se seleccionó un estudio con enfoque cualitativo para este estudio porque puede proporcionar un examen profundo e intensivo y, la comprensión de los múltiples aspectos de un fenómeno en un entorno natural (Quinn Patton y Cochran, 2007). Además, puede permitir una comprensión holística y más completa del contexto significativo (Punch, 2005), porque puede revelar las ideas y opiniones de las personas involucradas en el caso estudiado, que no pueden considerarse sin el contexto (Yardley, 2017). En este estudio, se examinaron cada uno de los discursos del alumnado y la adquisición de competencias 
Impacto de una experiencia Flipped Classroom en las percepciones sobre el aprendizaje del alumnado de Ciencias de la Actividad Física y el Deporte

en una experiencia de aprendizaje en la asignatura de Biomecánica del Movimiento Humano a través del aula invertida.

\section{Participantes}

El grupo de estudio de la presente investigación consta de 54 estudiantes de segundo año (22 mujeres, 32 hombres) de la Universidad de Granada (Campus de Melilla), de la Facultad de Ciencias de la Educación. El estudio se realizó en el segundo semestre del año académico 2020-2021. Durante 18 semanas lectivas, los contenidos teóricos y prácticos de la asignatura de Biomecánica del Movimiento Humano se organizaron para la preparación y visualización previa por parte del alumnado antes de llegar al aula para, una vez encontrarse en el aula se pudiera trabajar sobre los contenidos de manera grupal. La recogida de datos, se realizó a través de un diario personal del alumnado cumplimentando seis preguntas durante todo el periodo lectivo, contestando a las preguntas sobre adquisición de competencias y satisfacción educativa. Un ejemplo de las preguntas recogidas en los diarios reflexivos fue: 1) ¿Cómo valoras tu aprendizaje sobre los contenidos de esta semana? 2) ¿Cómo ha sido la organización y el trabajo en grupo durante la semana? y 3) ¿Qué competencias consideras que has aprendido esta semana y a través de qué dinámicas? En este contexto aparece la evaluación formativa tiene como objetivo generar la mejora y el proceso de aprendizaje de tres formas: (a) Optimizando el proceso de aprendizaje y la producción de los estudiantes; (b) Mejorando las prácticas docentes; (c) Realineando las aulas durante y después del desarrollo del proceso de enseñanza de la asignatura (Pérez-Pueyo et al., 2017). Este proceso de mejora y optimización se lleva a cabo desde una perspectiva humana. En este modelo, la utilización correcta del feedback es un punto clave (López et al., 2020). El rol del docente fue el de guía durante todo el proceso de recogida de datos, basando la evaluación en un proceso formativo, ofreciendo feedback al alumnado cada semana de cómo se estaban realizando cada uno de los diarios reflexivos. Dicho control de los diarios se hizo a través de comentarios y tutorías sobre un documento Excel compartido con el profesor de la asignatura y creado directamente mediante la herramienta de Formularios de Google. El feedback ofrecido semanalmente por el docente a aquel alumnado que no estuviera interiorizando adecuadamente las prácticas y contenidos, sirvió para guiar el aprendizaje y profundizar sobre él. Además, se profundizó sobre los discursos de los participantes a través de entrevistas semiestructuradas a ocho de los alumnos. Se informó a los participantes sobre la confidencialidad y que los datos de los diarios reflexivos y entrevistas se utilizarían únicamente con fines de investigación.

\section{Procedimiento}

Mediante un análisis detallado de los diarios reflexivos y la entrevista semiestructurada (Creswell, 2013), el objetivo del presente estudio fue profundizar en cómo los estudiantes de segundo año perciben su experiencia de aprendizaje en un aula invertida en contraste con el aprendizaje en una metodología tradicional y lo que destacan como importante para su experiencia de aprendizaje. Se desarrolló un documento con preguntas abiertas y flexibles para la entrevista (Tabla 1), con el objetivo de tener acceso a las opiniones, la comprensión y las experiencias de los estudiantes (Silverman y Patterson, 2014) para profundizar y ampliar la información recogida sobre las percepciones del alumnado con preguntas vinculadas y relacionadas con las que habían ido describiendo cada semana en el diario reflexivo. El primer autor recopiló y organizó individualmente las transcripciones en audio de las entrevistas de los participantes al final del segundo semestre y antes de su examen final. Uno de los investigadores transcribió cada diario con Nvivo (Version 12, QSR International Pty Ltd, Melbourne, Australia), sumando hasta 216 páginas entre ambos métodos usados en la recolección de datos.

La opción metodológica para el análisis es mixta, al partir de las cuestiones previas marcadas en el guion de la entrevista semiestructurada y los diarios reflexivos (deductiva) e inductiva, de tipo emergente, al respetar los discursos de los participantes. Se han utilizado algunas de las pautas descritas en la Teoría Fundamentada, trazando los discursos de la investigación desde lo particular a lo general, mediante el proceso de categorización y posterior codificación (Glaser et al., 2019). Generalmente causado por los propios datos, en este caso, del discurso del alumnado participante (Sandín Esteban, 2000). Se ha realizado una codificación axial; abierta, a partir de las categorías previas y emergente, fundamentalmente en el segundo y tercer nivel de categorización, desde la interpretación de los 
investigadores. Para ello, se partió de los discursos de los participantes e ir desarrollando el árbol de categorías, que, posteriormente, se fue completando y codificando con nuevas lecturas de los diarios reflexivos y las entrevistas realizadas.

\section{Tabla 1}

\section{Guía sobre las preguntas de la entrevista semiestructurada}

1. Ahora que conoces Flipped Classroom ¿Prefieres una metodología de enseñanza tradicional o de aula invertida?

2. ¿Qué lecciones has aprendido de tu participación en la asignatura mediante la metodología Flipped Classroom?

3. ¿Crees que esta metodología ha sido útil para tu aprendizaje formando parte de un grupo? ¿Por qué?

4. ¿Ha aumentado tu motivación para aprender e involucrarte más con dicha metodología?

5. ¿Has sido capaz de llevar un ritmo propio de aprendizaje y gestionar mejor el tiempo de dedicación?

6. ¿Se ha aumentado el nivel de competencia en cuanto a la aplicación de los contenidos aprendidos en las sesiones previas al trabajo en clase?

7. ¿Qué tipo de habilidades y competencias crees que has adquirido a lo largo del semestre con esta metodología?

8. En su opinión, ¿qué tal ha sido la relación con el profesor durante el transcurso de la asignatura?

Los datos de la entrevista se analizaron mediante análisis de contenido de manera no lineal, es decir, el proceso de análisis se realizó mediante un proceso de ida y vuelta en el tratamiento de los datos (Miles y Huberman, 1994). El análisis de contenido es un método flexible para trabajar con datos de texto y es adecuado cuando el objetivo es obtener puntos de vista y percepciones de los participantes (Creswell, 2013). Usando codificación emergente, los autores establecieron categorías después de un examen preliminar de los datos (Silverman y Patterson, 2014). Más tarde, fueron comparadas ambas listas de categorías, discutiendo las diferencias y ajustando y creando el árbol de categorías final. Posteriormente se realizó una comparación final, comprobando la confiabilidad de la codificación. La confiabilidad de las interpretaciones se desarrolló a través de varias discusiones entre los autores, desarrollando así la reflectividad en el proceso.

En cuanto al análisis de los datos, apoyado por el software NVivo, se ha asentado principalmente en las matrices de codificación, para hacer surgir las relaciones entre las diferentes categorías y la referencia cruzada para relacionar las categorías que importan en cada momento del análisis y los atributos otorgados a los participantes. Esto, ha permitido confrontar, crear relaciones y hacer visibles los interrogantes formados a partir de los discursos de los participantes. La estrategia utilizada nos permite enfrentar las teorías implícitas de los participantes con las de los investigadores, dando como resultado la teoría sustantiva de la investigación (Trigueros Cervantes et al., 2016)

\section{Consideraciones éticas}

Todos los estudiantes de este estudio fueron tratados de acuerdo con las pautas de la Asociación Americana de Psicología (APA), que aseguraron el anonimato de las respuestas de los participantes. El estudio se llevó a cabo de acuerdo con los principios éticos de la declaración de Helsinki de 1964 para la investigación en humanos y los criterios éticos y de buenas prácticas establecidos en investigación por parte de la Universidad de Granada. Siguiendo a Quinn Patton y Cochran (2007), se han tenido en cuenta las cuestiones éticas que deben seguirse en toda investigación y que son la confidencialidad y el consentimiento. Por ello, el alumnado participante fue debidamente informados sobre los objetivos y temas a tratar y se preservó su identidad por encima de todo. Cada una de las intervenciones realizadas por los participantes fue descrita en el texto con un pseudónimo que ellos mismos eligieron. 
Impacto de una experiencia Flipped Classroom en las percepciones sobre el aprendizaje del alumnado de Ciencias de la Actividad Física y el Deporte

\section{Rigor}

El compromiso y el rigor científico se han seguido mediante un compromiso profundo con las transcripciones, con el tema, que incluye una recopilación exhaustiva de datos, opinión de expertos, análisis de las redacciones del alumnado, verificación de los métodos empleados para realizar un análisis detallado y profundo y las notas durante todo el semestre sobre las clases de la asignatura que guían el proceso de análisis e interpretación de los datos. El tratamiento y análisis de los datos se revisó en cada uno de sus pasos por parte de dos de los investigadores implicados en la investigación.

\section{Resultados y discusión}

El objetivo del presente estudio es el de conocer los niveles de satisfacción y adquisición de competencias por parte del alumnado tras la inmersión en una experiencia de enseñanza-aprendizaje basada en la metodología de aula invertida. A partir del análisis, encontramos siete temas que se repitieron en el material de datos. En esta sección los presentamos y discutimos como nuestros principales resultados. Los seis temas son:

- Compromiso con los compañeros

- Aprender con compañeros

- Relación con el profesor

- Entorno de aprendizaje físico

- Usar videos para aprender contenido nuevo

- Ser reconocido

La pregunta inicial en los diarios reflexivos fue qué forma de aprendizaje preferían los estudiantes en general, y una gran mayoría de los participantes dijeron que preferían aprender en el aula invertida a aprender cómo han venido vivenciando en el resto de asignaturas. De los 54 participantes, 49 prefirieron el aula invertida a las clases tradicionales magistrales, mientras que tres participantes prefirieron la enseñanza magistral y dos no destacaron un modo de enseñanza sobre el otro. Creemos que los siete temas identificados a través del análisis pueden ayudar a comprender por qué los estudiantes favorecieron el aprendizaje en una metodología basada en aula invertida.

El hecho de que las dimensiones psicosociales influyan en la participación de los estudiantes es importante para comprender las experiencias de aprendizaje del alumnado y, en consecuencia, para el aprendizaje y los logros adquiridos (Awidi y Paynter, 2019). A continuación, se discuten estos temas, utilizando extractos del material de los diarios para ilustrar las reflexiones de los estudiantes. Los resultados se discuten como temas separados; Sin embargo, observamos que la riqueza de los datos cualitativos implica que algunos temas pueden superponerse parcialmente entre sí.

\section{Compromiso con los compañeros}

El compromiso con los compañeros implica que los estudiantes estén dispuestos a invertir tiempo y esfuerzo con sus compañeros y, como mostró nuestro análisis, esto fue particularmente evidente en relación con la participación grupal. En línea con Cheng et al. (2019), se descubrió que el compromiso con los compañeros era un tema recurrente cuando los estudiantes hablaban sobre el aula invertida. La participación en el aprendizaje grupal fue voluntaria durante las actividades de aprendizaje y muchos participantes expresaron un sentido de compromiso para contribuir al grupo en el aula invertida. En este contexto y en concordancia con los hallazgos encontrados por Steen-Utheim y Foldnes (2018), se detalla la aportación de López (7) "La asistencia a algunas clases era opcional, pero no se sentía como tal ya que te sientes parte del grupo y obligado a venir a la sesión para trabajar en equipo" o, en la misma línea, "esta modalidad me hizo participar en el trabajo en grupo. Fue más fácil venir a clase gracias al grupo. Vas a tu grupo y luego todos los miembros deben contribuir" (Martín, 43).

Además, los estudiantes destacan cómo el compromiso con el grupo contribuyó a un entorno de aprendizaje social positivo, con una responsabilidad compartida en el grupo, tanto en la asignatura como 
con los compañeros: Consuegra (32), por ejemplo, reflexiona sobre cómo el trabajo en grupo desencadenó una responsabilidad dentro de él, tanto en relación con el tema como con sus compañeros:

“...hicimos un esfuerzo, ya que no solo dependía de cada uno de nosotros individualmente, sino como grupo. Se formó una comunidad, fue divertido venir y conocer gente. Y las clases se volvieron divertidas. Fue agradable trabajar juntos, y después de un tiempo nos conocimos y comenzamos a profundizar más y más. Realmente había un sentido de comunidad en torno a la asignatura".

Tener un sentido de pertenencia al grupo, como lo expresa el alumnado del presente estudio es una de las dimensiones que Masika y Jones (2016) presentan como aspecto influyente en el compromiso de los estudiantes. El compromiso que estos estudiantes experimentan en el modelo de aula invertida influye en su inversión e involucración en el grupo, y cuando el alumnado también expresa entusiasmo por los contenidos desarrollados en la asignatura, descrito por Murillo-Zamorano et al. (2019), como la dimensión afectiva del compromiso.

\section{Aprender con compañeros}

El alumnado enfatizó sobre el aprendizaje en grupos como algo importante para su experiencia de aprendizaje. Intervenciones que irían en concordancia con las mostradas en diversos estudios (Busebaia y John, 2020; Steen-Utheim y Foldnes, 2018). Las respuestas indican que la participación en grupos apoya su aprendizaje y compromiso, principalmente debido a las posibilidades de hacer preguntas, discutir y colaborar con compañeros. En el aula invertida, había más posibilidades de aprendizaje en grupo, dado que las sesiones de clase se organizaban con mucho tiempo para el trabajo posterior en los grupos fijos. Siguiendo la aportación de Hisham (52):

"[En el aula invertida] hicimos muchas preguntas dentro del grupo. Si un planteamiento era complicado, no nos dábamos por vencidos hasta que cada miembro del grupo decía: "Sí, lo entendí, lo entiendo". Estaba bien repasar la tarea varias veces, si era necesario. Por lo general, uno del grupo tomaba las riendas en la organización de la búsqueda de información y a partir de ahí nos poníamos a trabajar en equipo. Esto sin duda ha favorecido el aprendizaje e implicación de todos."

El papel de la contribución de los demás al propio aprendizaje, se destaca en la aportación de la alumna Ros (41), en ella se destaca el aprendizaje entre pares, como lo ejemplifica ella misma, potencia el aprendizaje de los estudiantes porque la contribución de los diferentes niveles de competencias de los demás respalda el crecimiento individual. La experiencia de aprendizaje llega a cada uno de los miembros del grupo cuando las debilidades y fortalezas de unos y otros se complementan y favorecen el desarrollo competencial de todos los participantes (Chen, 2021).

En el siguiente extracto, Lebrón (7) explica cómo tiene lugar su comprensión en el espacio de aprendizaje social, después de ayudar primero al grupo con una búsqueda por su cuenta: "Fue muy agradable esos momentos en que pude explicar algo a los demás en el grupo, me hizo sentir bien. Además, aprendes por ti mismo tratando de explicarles a tus compañeros cómo entenderlo."

Tanto Ros como Lebrón, describen cómo se involucran tanto en la actividad cognitiva individual como en la interacción con sus compañeros, un punto central en el punto de vista sociocultural sobre el aprendizaje (Lin, 2019). Consideramos que los sentimientos de compromiso, seguridad y reconocimiento que se experimentan en las metodologías activas de enseñanza-aprendizaje tienen valor pedagógico. Nuestros hallazgos indican que estos sentimientos están relacionados con el proceso de participación y aprendizaje grupal. Datos que irían en concordancia con los mostrados por Steen-Utheim y Foldnes (2018) El análisis también ilustró que el desarrollo del conocimiento surgió a través de la contribución de otros durante la participación grupal.

\section{Relación con el profesor}

La relación con el instructor se percibe de manera diferente si el alumnado compara los dos modos de aprendizaje. Muchos estudiantes informaron un contacto más cercano con el profesor en el aula invertida que en el modelo de enseñanza tradicional (Almanasef et al., 2020; Jdaitawi, 2020). Algunos/as estudiantes comentan que este contacto es algo que extrañan de la educación secundaria. En esta línea, encontramos la aportación de Al Luch (25): 
Impacto de una experiencia Flipped Classroom en las percepciones sobre el aprendizaje del alumnado de Ciencias de la Actividad Física y el Deporte

"Fue más fácil ponerse en contacto con el profesor en el modo de aula invertida. El profesor interactúa mucho más, nosotros nos sentamos a trabajar y él se acerca a nosotros. Entre mis profesores, este es el que mejor conozco y más confianza tengo [el instructor del aula invertida]. Los otros profesores, que solo dan la clase con PowerPoint, simplemente dan el tema y se marchan. Es algo que extraño de la educación secundaria, un contacto más cercano con los profesores. Te da el valor para hacer preguntas."

Siguiendo con las mismas sensaciones, otra alumna detallaba: "Me gustó la disponibilidad del profesor en contraste con dar la clase a muchos estudiantes que solo escuchan. Siento que se puede dominar mejor el tema, porque te sientes más seguro y tienes ganas de venir a clase." (Roldán, 38).

Las posibilidades que ofrece el uso de metodologías activas para la interacción con el profesor parecen más fáciles en el aula invertida según el alumnado participante. El contacto con el profesor que establece el alumnado, también tiene un efecto positivo en un contexto social más amplio (Yoon et al., 2020). Siguiendo a Gómez-Carrasco et al. (2019), condiciones favorecedoras de la participación y motivación del alumnado influyen en la mejora del aprendizaje de los participantes.

\section{Entorno de aprendizaje físico}

Para muchos estudiantes, el entorno físico de aprendizaje en la clase tradicional se percibe como un obstáculo para establecer una relación con el profesor (Almanasef et al., 2020). La organización de la clase, en combinación con el tamaño de la clase reduce las posibilidades de los estudiantes de interactuar con el profesor y con sus compañeros. Otros estudiantes también señalan obstáculos organizativos por los que se sienten limitados cuando se anima a los estudiantes a trabajar en grupos sobre las diferentes actividades.

En algunas clases te sientas en largas filas. Es difícil formar grupos allí como lo hicimos en el aula invertida. (Hierro, 10). Te sientas en un espacio reducido, con apenas espacio para tu cuaderno. No hay posibilidad de movimiento en las inmediaciones. Si un compañero de estudios quiere explicar las cosas, no hay espacio para explicar. (Romacho, 19). En cuanto a la facilidad de interacción debido a la organización y material dentro del aula encontramos la aportación de Ferreri (40):

"[En Flipped] pasó que algunos de nosotros queríamos explicar algún concepto a los compañeros, y estábamos sentados en la clase en grupos donde había una pequeña pizarra. Nos pusimos de pie y escribimos en la pizarra, juntos, y resolvimos la actividad juntos de esa manera."

Estos discursos ilustran cómo el entorno de aprendizaje físico restringe la participación de los participantes. El comportamiento de los estudiantes, que se muestra como tiempo y esfuerzo, interacción y participación (Masika yJones, 2016), está restringido por su entorno físico de aprendizaje. Como señalan Hierro y Romacho, en la organización de la clase tradicional, las condiciones para la participación son limitadas y la explicación e interacción son difíciles. Sin embargo, en el aula invertida, las áreas de enseñanza son más flexibles, lo que permite que los grupos establezcan su propio espacio. Ferreri también señala cómo los estudiantes utilizan las herramientas disponibles en la sala de grupo para sus actividades de aprendizaje colaborativo. Aportaciones que irían en línea con las encontradas por Jdaitawi (2020) sobre el aumento de la participación del alumnado en las metodologías de enseñanza-aprendizaje activas.

En la definición de aula invertida que ofrecen Abeysekera y Dawson (2015), el aprendizaje social juega un papel central. Visto desde una perspectiva sociocultural, el papel de la contribución de los demás al propio aprendizaje es de suma importancia. Con base en el presente estudio, y el de Foldnes (2016), organizar sesiones de clase con aprendizaje colaborativo en grupos fijos parece un tipo de aprendizaje social particularmente prometedor, que puede resultar en sentimientos elevados de compromiso, reconocimiento personal y por parte del profesor y compañeros, que creemos son beneficioso para el aprendizaje. El aula basada en una estructura y organización tradicional pareció establecerse con menos oportunidades para el aprendizaje en grupo, por lo tanto, se mostró un apoyo estructural más fuerte para el aprendizaje en grupo en las metodologías activas de enseñanza-aprendizaje.

\section{Usar vídeos para aprender un contenido nuevo}

En general, los estudiantes informaron estar muy satisfechos con el uso de videos. Los estudiantes mencionaron varias razones en esta línea. Una era la posibilidad de pausar y volver a reproducir los videos. 
"Tomo notas mientras veo los videos. Dejo el video pausado y aclaro términos. Con los videos es bueno poder escribir a tu propia velocidad y entender mejor los conceptos." (Polo, 42). En este contexto, otro alumno recalca la posibilidad de poder ver varias veces y en varios momentos los vídeos para entender mejor los contenidos explicados: "en un video, puedes simplemente rebobinar y volver a ver varias veces. En una clase tradicional no es posible pausar la clase y ajustarla al ritmo individual (Silva, 13).

Los videos permiten autorregular el propio aprendizaje, porque los estudiantes se pueden ajustar al contenido pausando y rebobinando. Esta flexibilidad, para pausar y ver los videos cuando ellos puedan, permite a los estudiantes un aprendizaje a su propio ritmo porque pueden ajustar este ritmo según sus necesidades. El uso de estrategias para dominar el conocimiento al visualizar los videos, como ilustran los extractos de Polo y Silva, refleja su compromiso cognitivo, crucial para el desarrollo del conocimiento del individuo y se desarrollan a través de la interacción con uno mismo y con los demás. En el aula tradicional, sin embargo, el profesor continúa con el tema, prestando poca atención a los ritmos de aprendizaje del alumnado. En consecuencia, esto podría influir en el desarrollo cognitivo de los participantes y, por lo tanto, ser un obstáculo para el aprendizaje posterior.

Además, en línea con Masika y Jones (2016), se encontró una preferencia más fuerte por la enseñanza de aula invertida, lo que puede deberse a los recursos en línea y el aprendizaje autodirigido que facilitan la capacidad de volver a visitar el contenido varias veces (especialmente con conceptos o contenidos de mayor dificultad) y la organización en clases activas proporcionando más interacción de modo que los compañeros puedan explicar el contenido de diferentes maneras, facilitando posteriormente un mejor contexto de aprendizaje y mayor comprensión por parte del alumnado (Murillo-Zamorano et al., 2019).

\section{Ser reconocido}

Es importante que los estudiantes sean reconocidos como individuos y pasen de ser un número más en el sistema educativo universitario, tanto por sus compañeros como por su profesor especialmente. El reconocimiento por parte de los compañeros se menciona con mucha frecuencia cuando se habla del aula invertida, donde los grupos se sientan juntos durante todo el semestre. Este reconocimiento también implica compromiso:

“..., lo que se espera de ti es más visible. Esto te hace querer tener un buen desempeño en grupo, no quieres parecer como alguien que no puede resolver problemas. En una clase tradicional puedes esconderte, pero en un aula invertida no. Muchos estudiantes necesitamos que nos planteen estos escenarios para ser vistos y estar motivados". (Barrueco, 14)

Algunos participantes también destacan el reconocimiento por parte del profesor. En esta línea es la aportación de Ros (41), "Flipped te hace más cercano. Un día el profesor me comentó "no viniste la semana pasada, ¿pasó algo?", nunca me lo habían dicho, parece que daba igual y luego te sientes más comprometida de venir a clase". Se observa, como un simple gesto de atención y preocupación por parte del profesorado, mantiene el compromiso del alumnado.

Las relaciones entre el estudiante y el profesor, son descritas y mostradas por Ros. Estas influencias psicosociales en la participación del alumnado son importantes, la participación no se trata solo del "estado estático interno" de un estudiante, si no que tiene en cuenta la responsabilidad y la implicación (Yoon et al., 2020). La experiencia del individuo está inmersa en el contexto sociocultural y está influenciada por factores institucionales e individuales, como el papel del profesor y su relación con el alumnado. El papel del profesor en la motivación del alumnado y alentar las actividades en clase proporcionando un ambiente en el aula estimulante, podría crear mejores interacciones entre los estudiantes y la probabilidad de obtener mejores resultados de aprendizaje por parte del alumnado participante (Busebaia y John, 2020).

\section{Conclusiones}

En este estudio, se han analizado las percepciones de los estudiantes sobre su experiencia de aprendizaje en un aula invertida. En general, los estudiantes informan de una experiencia de aprendizaje más positiva en el aula invertida. Los estudiantes enumeran una variedad de razones para esto, pero un 
Impacto de una experiencia Flipped Classroom en las percepciones sobre el aprendizaje del alumnado de Ciencias de la Actividad Física y el Deporte

vínculo común son las posibilidades de participación que implica el aula invertida. Una de las principales conclusiones extraídas del presente estudio es que la dimensión afectiva se estimula especialmente en el aula invertida, en comparación con el aula tradicional. Por ejemplo, los estudiantes informaron repetidamente de un sentimiento de compromiso con sus compañeros y el aumento de la relación con el profesor. Otros temas emergentes que se relacionan con el afecto de los estudiantes son las nociones de sentirse seguro y ser reconocido. Los resultados también muestran que la dimensión cognitiva del compromiso se mostró más claramente en el aprendizaje de aula invertida, y los estudiantes aprendieron más de sus compañeros.

Una implicación importante de estos hallazgos es la sugerencia de que las aportaciones de los/as estudiantes ponen de manifiesto y representan una inversión más fuerte en la estrategia del aula invertida que tiene ventajas posteriores para los resultados del aprendizaje. Se pueden hacer dos recomendaciones a partir de estos hallazgos: (a) que hay que seguir investigando en si estos aspectos se desempeñan mejor en un entorno de aula invertida además de ser preferido por los estudiantes y (b) que estos aspectos deben tenerse muy en cuenta al diseñar un entorno de aula invertida. Los discursos del alumnado han mostrado las preferencias de los estudiantes por el entorno de enseñanza de aula invertida sobre un entorno de enseñanza tradicional. Sin embargo, debido a la novedad del aprendizaje centrado en el estudiante, muchos de ellos pueden mostrar cierta resistencia al enfoque de enseñanza invertida. Esto podría resultar en actitudes más bajas hacia las actividades del curso, la creencia de que el curso está desorganizado y la creencia más débil en su capacidad para completar el trabajo establecido. Además, a pesar de que algunos estudios sugieren que algunos estudiantes se inclinarán a resistir el ambiente de aula invertida debido a sus preferencias por aprender en clase, en lugar de antes de la clase. Esto no debería disuadir a los docentes de cambiar sus estrategias y planificar adecuadamente.

En cuanto a las limitaciones del estudio, sería importante comprender la perspectiva por parte del profesorado participante con el objetivo de enriquecer y enlazar las aportaciones del alumnado.

Contribución de cada Autor: Conceptualización, Ricardo Martín-Moya y Encarnación Ruiz-Montero; Metodología, Ricardo Martín-Moya y Pedro Jesús Ruiz-Montero; Análisis, Ricardo Martín-Moya y Augusto Hoyo-Guillot; Escritura del manuscrito, Ricardo Martín-Moya, Augusto Hoyo-Guillot, Encarnación RuizMontero y Pedro Jesús Ruiz-Montero; Escritura, Revisión y Edición, Ricardo Martín-Moya y Pedro Jesús RuizMontero; Supervisión, Ricardo Martín-Moya".

Financiación: Esta investigación no recibió ninguna financiación externa

Conflicto de Intereses: Las/os autoras/es declaran que no tienen conflicto de intereses.

\section{Referencias}

Abeysekera, L., y Dawson, P. (2015). Motivation and cognitive load in the flipped classroom: definition, rationale and a call for research. Higher Education Research and Development, 34(1). https://doi.org/10.1080/07294360.2014.934336

Almanasef, M., Almaghaslah, D., Portlock, J., y Chater, A. (2020). Qualitative investigation of the flipped classroom teaching approach as an alternative to the traditional lecture. Pharmacy Education, 20. https://doi.org/10.46542/pe.2020.201.p142-150

Aranda, A. F., Herguedas, J. L. A., Dieste, S. A., y Martín, R. R. (2018). La evaluación formativa de las competencias genéricas en la formación del profesorado de educación física. Estudios Pedagógicos, 44(2).

Assmann, H. (2002). Placer y ternura en la educación. Hacia una sociedad aprendiente. Narcea, 1(4).

Awidi, I. T., y Paynter, M. (2019). The impact of a flipped classroom approach on student learning experience. Computers and Education, 128. https://doi.org/10.1016/j.compedu.2018.09.013

Bachnak, R., y Maldonado, S. C. (2014). A flipped classroom experience: Approach and lessons learned. ASEE Annual Conference and Exposition, Conference Proceedings. https://doi.org/10.18260/1-2$-19942$ 
Baepler, P., Walker, J. D., y Driessen, M. (2014). It's not about seat time: Blending, flipping, and efficiency in active learning classrooms. Computers and Education, 78. https://doi.org/10.1016/j.compedu.2014.06.006

Bishop, J. L. (2013). A controlled study of the flipped classroom with numerical methods for engineers. ProQuest Dissertations and Theses.

Bretón de la Loza, M. M., Zúñiga Hernández, O. Y., Terrazas Meraz, M. A., Garduño Díaz, S. D., Guerrero Ceh, J. G. y Carranco Gómez, S. (2021). Methodological model for the development of clinical competencies in nutrition students. International Journal of New Education, 8, 173-187.

Busebaia, T. J. A., y John, B. (2020). Can flipped classroom enhance class engagement and academic performance among undergraduate pediatric nursing students? A mixed-methods study. Research and Practice in Technology Enhanced Learning, 15(1). https://doi.org/10.1186/s41039-020-01241

Canales-Ronda, P., y Hernández-Fernández, A. (2019). Metodología flipped classroom en la enseñanza universitaria TT - Flipped Classroom Methodology in University Teaching TT - Metodología flipped classroom no ensino universitário. Revista Iberoamericana de Educación Superior, 10(28).

Casey, A. (2014). Models-based practice: Great white hope or white elephant? Physical Education and Sport Pedagogy, 19(1). https://doi.org/10.1080/17408989.2012.726977

Chen, C. C. (2021). Effects of flipped classroom on learning outcomes and satisfaction: An experiential learning perspective. Sustainability, 13(16). https://doi.org/10.3390/su13169298

Cheng, L., Ritzhaupt, A. D., y Antonenko, P. (2019). Effects of the flipped classroom instructional strategy on students' learning outcomes: a meta-analysis. Educational Technology Research and Development, 67(4). https://doi.org/10.1007/s11423-018-9633-7

Creswell, J. (2013). Research Design: Qualitative, quantitative, and mixed methods approach. Author.

Davies, R. S., Dean, D. L., y Ball, N. (2013). Flipping the classroom and instructional technology integration in a college-level information systems spreadsheet course. Educational Technology Research and Development, 61(4). https://doi.org/10.1007/s11423-013-9305-6

Fidalgo-Blanco, A., Sein-Echaluce, M. L., y García-Pẽalvo, F. J. (2019). Impact indicators of educational innovations based on active methodologies. Pervasive Health: Pervasive Computing Technologies for Healthcare. https://doi.org/10.1145/3362789.3362894

Foldnes, N. (2016). The flipped classroom and cooperative learning: Evidence from a randomised experiment. Active Learning in Higher Education, 17(1). https://doi.org/10.1177/1469787415616726

Gallardo Fuentes, F., López Pastor, V. M., y Carter Tuhillier, B. (2018). Efectos de la Aplicación de un Sistema de Evaluación Formativa en la Autopercepción de Competencias Adquiridas en Formación Inicial del Profesorado. Estudios Pedagógicos, 44(2). https://doi.org/10.4067/s071807052018000200055

Glaser, B. G., Strauss, A. L., Glaser, B. G., y Strauss, A. L. (2019). The Discovery of Grounded Theory. The Discovery of Grounded Theory. https://doi.org/10.4324/9780203793206-1

Gómez-Carrasco, C. J., Monteagudo-Fernández, J., Sainz-Gómez, M., y Moreno-Vera, J. R. (2019). Effects of a gamification and flipped-classroom program for teachers in training on motivation and learning perception. Education Sciences, 9(4). https://doi.org/10.3390/educsci9040299

Hermsen, J. L., Mokadam, N. A., y Verrier, E. D. (2019). Flipping the Classroom: How to Optimize Learning in the Didactic Setting. Thoracic surgery clinics 29(3). https://doi.org/10.1016/j.thorsurg.2019.04.002

Higueras-Rodríguez, L., Medina-García, M., y Molina-Ruiz, E. (2020). Analysis of courses and teacher training programs on playful methodology in Andalusia (Spain). Education Sciences, 10(4). https://doi.org/10.3390/educsci10040105

Howell, D. (2013). Effects of an inverted instructional delivery model on achievement of ninth-grade physical science honors students. Gardner-Webb University.

Hung, H. T. (2015). Flipping the classroom for English language learners to foster active learning. Computer Assisted Language Learning, 28(1). https://doi.org/10.1080/09588221.2014.967701 
Impacto de una experiencia Flipped Classroom en las percepciones sobre el aprendizaje del alumnado de Ciencias de la Actividad Física y el Deporte

Jdaitawi, M. (2020). Does flipped learning promote positive emotions in science education? A comparison between traditional and flipped classroom approaches. Electronic Journal of ELearning, 18(6). https://doi.org/10.34190/JEL.18.6.004

Jeong, J. S., González-Gómez, D., y Cañada-Cañada, F. (2021). How does a flipped classroom course affect the affective domain toward science course? Interactive Learning Environments, 29(5). https://doi.org/10.1080/10494820.2019.1636079

Landi, D., Fitzpatrick, K., y McGlashan, H. (2016). Models based practices in physical education: A sociocritical reflection. Journal of Teaching in Physical Education, 35(4). https://doi.org/10.1123/jtpe.2016-0117

Lin, Y. T. (2019). Impacts of a flipped classroom with a smart learning diagnosis system on students' learning performance, perception, and problem-solving ability in a software engineering course. Computers in Human Behavior, 95. https://doi.org/10.1016/j.chb.2018.11.036

López, V. M., Soria, M. M., Arias, C. P., Carlos, J., y Arribas, M. (2020). La importancia de utilizar la Evaluación Formativa y Compartida en la Formación Inicial del Profesorado de Educación Física: los Proyectos de Aprendizaje Tutorado como ejemplo de buena práctica. Retos: Nuevas Tendencias En Educación Física, Deporte y Recreación, 37, 620-627.

Marlowe, C. A. (2012). The effect of the flipped classroom on student achievement and stress. Journal of Chemical Information and Modeling, 53.

Masika, R., y Jones, J. (2016). Building student belonging and engagement: insights into higher education students' experiences of participating and learning together. Teaching in Higher Education, 21(2). https://doi.org/10.1080/13562517.2015.1122585

Matsumoto, T. (2016). The Flipped Classroom Experience of Gamified. Creative Education, 07(10). https://doi.org/10.4236/ce.2016.710152

McNally, B., Chipperfield, J., Dorsett, P., del Fabbro, L., Frommolt, V., Goetz, S., Lewohl, J., Molineux, M., Pearson, A., Reddan, G., Roiko, A., y Rung, A. (2017). Flipped classroom experiences: student preferences and flip strategy in a higher education context. Higher Education, 73(2). https://doi.org/10.1007/s10734-016-0014-z

Miles, M., y Huberman, M. (1994). Data management and analysis methods. Handbook of Qualitative Research.

Monereo, C., y Alvarez, C. E. I. (2013). Changing university teacher's identity: Training based on dramatized incidents | Cambiar la identidad docente en la universidad. formación basada en incidentes dramatizados. Infancia y Aprendizaje, 36(3).

Murillo-Zamorano, L. R., López Sánchez, J. Á., y Godoy-Caballero, A. L. (2019). How the flipped classroom affects knowledge, skills, and engagement in higher education: Effects on students' satisfaction. Computers and Education, 141. https://doi.org/10.1016/j.compedu.2019.103608

Murphree, D. S. (2014). "Writing wasn't really stressed, accurate historical analysis was stressed ": Student Perceptions of In-Class Writing in the Inverted, General Education, University History Survey Course. The History Teacher, 47(2).

O'Flaherty, J., y Phillips, C. (2015). The use of flipped classrooms in higher education: A scoping review. Internet and Higher Education, 25. https://doi.org/10.1016/j.iheduc.2015.02.002

Pérez-Pueyo, A., López-Pastor, V., Hortigüela, D., Gutiérrez-García, C., Casado, O., Hernando, A., Lorente-Catalán, E., Alonso-Cortés, D., Diez-Fernández, Á., Domínguez-Fernández, R., González-Alonso, I., Fernández-Fernández, R., García-González, M., Santos-Pastor, M., Martínez-Muñoz, L., Martínez-Mínguez, L., Heras, C., Leal, P., Herrán, I., ... Barba, J. (2017). Evaluación formativa y compartida en educación: experiencias de éxito en todas las etapas educativas. Universidad de León, Secretariado de Publicaciones.

Punch, K. F. (2005). Introduction to Social Research: Quantitative and Qualitative Approaches. SAGE. Quinn Patton, M., y Cochran, M. (2007). A Guide to Using Qualitative Research Methodology. Medecins Sans Frontieres, 1-36.

Sandín Esteban, M. (2000). Criterios de validez en la investigación cualitativa: de la objetividad a la solidaridad. Revista de Investigación Educativa, 18(1), 223-242. 
Silverman, R. M., y Patterson, K. L. (2014). Qualitative research methods for community development. Qualitative Research Methods for Community Development. https://doi.org/10.4324/9781315797762

Steen-Utheim, A. T., y Foldnes, N. (2018). A qualitative investigation of student engagement in a flipped classroom. Teaching in Higher Education, 23(3). https://doi.org/10.1080/13562517.2017.1379481

Strayer, J. F. (2012). How learning in an inverted classroom influences cooperation, innovation and task orientation. Learning Environments Research, 15(2). https://doi.org/10.1007/s10984-012-9108-4

Trigueros Cervantes, C., Rivera García, E., Moreno Doña, A., y Muñoz Luna, R. (2016). Uso del software CAQDAS Nvivo en ciencias sociales: Para la investigación con grupos de discusión. Index de Enfermeria, 25(3), 171-174.

van Alten, D. C. D., Phielix, C., Janssen, J., y Kester, L. (2020). Effects of self-regulated learning prompts in a flipped history classroom. Computers in Human Behavior, 108. https://doi.org/10.1016/j.chb.2020.106318

Wijayati, N., Sumarni, W., y Supanti, S. (2019). Improving Student Creative Thinking Skills Through Project Based Learning. KnE Social Sciences. https://doi.org/10.18502/kss.v3i18.4732

Yardley, L. (2008). Demonstrating validity in qualitative psychology. Qualitative psychology: A practical guide to research methods, 2, 235-251. https://doi.org/10.1080/17439760.2016.1262624

Yoon, S., Kim, S., y Kang, M. (2020). Predictive power of grit, professor support for autonomy and learning engagement on perceived achievement within the context of a flipped classroom. Active Learning in Higher Education, 21(3). https://doi.org/10.1177/1469787418762463 